\title{
Extensibility of Human Asteroid Mission to Mars and Other Destinations
}

\author{
Mark A. McDonald ${ }^{1}$, Jose M. Caram ${ }^{2}$, Pedro Lopez ${ }^{3}$, Heather D. Hinkel ${ }^{4}$, Jonathan T. Bowie ${ }^{5}$, \\ Paul A. Abell ${ }^{6}$, Bret G. Drake ${ }^{7}$, Roland M. Martinez ${ }^{8}$ \\ Johnson Space Center, Houston, TX, 77058 \\ Paul W. Chodas ${ }^{9}$ \\ Jet Propulsion Laboratory, Pasadena, CA, 91109 \\ Kurt Hack ${ }^{10}$ \\ Glenn Research Center, Cleveland, $\mathrm{OH} 44135$ \\ Daniel D. Mazanek ${ }^{11}$ \\ Langley Research Center, Hampton, VA, 23681
}

\begin{abstract}
This paper will describe the benefits of execution of the Asteroid Redirect Mission as an early mission in deep space, demonstrating solar electric propulsion, deep space robotics, ground and on-board navigation, docking, and EVA. The paper will also discuss how staging in trans-lunar space and the elements associated with this mission are excellent building blocks for subsequent deep space missions to Mars or other destinations.
\end{abstract}

\section{Nomenclature}

$\begin{array}{ll}\text { ARM } & =\text { Asteroid Redirect Mission } \\ A R C M & =\text { Asteroid Redirect Crewed Mission } \\ \text { ARRM } & =\text { Asteroid Redirect Robotic Mission } \\ A R R V & =\text { Asteroid Redirect Robotic Vehicle } \\ D O F & =\text { Degree of Freedom } \\ D R O & =\text { Distant Retrograde Orbit } \\ D S S & =\text { Deployable Space Systems } \\ E H F & =\text { Extremely High Frequency } \\ E L V & =\text { Expendable Launch Vehicle }\end{array}$

${ }^{1}$ Lead, Advanced Mission Development Group, Technical Integration Office, Engineering Directorate, NASA Johnson Space Center/EA4

${ }^{2}$ Deputy Manager, Technical Integration Office, Engineering Directorate, NASA Johnson Space Center/EA4

${ }^{3}$ Aerospace Engineer, Advanced Mission Development Group, Technical Integration Office, Engineering Directorate, NASA Johnson Space Center/EA4

${ }^{4}$ Rendezvous Sensor Lead, Aeroscience and Flight Mechanics Division, Engineering Directorate, NASA Johnson Space Center/EG6

${ }^{5}$ AEMU SE\&I Lead Engineer, Space Suit and Crew Survival Systems Branch, Engineering Directorate, NASA Johnson Space Center/EC5

${ }^{6}$ Lead Scientist for Planetary Small Bodies, Astromaterials Research and Exploration Science Directorate, NASA Johnson Space Center/KR

${ }^{7}$ Deputy Manager, Exploration Missions and Systems Office, NASA Johnson Space Center/YX

${ }^{8}$ Aerospace Engineer, Exploration Mission Systems Office, Human Exploration Development Support, NASA Johnson Space Center/YX111

${ }^{9}$ Senior Engineer, NASA NEO Program Office, 301-121, Jet Propulsion Laboratory, California Institute of Technology.

${ }^{10}$ Aerospace Engineer, Mission Design and Analysis Branch, NASA Glenn Research Center, Cleveland, OH 44135, Senior Member AIAA.

${ }^{11}$ Senior Space Systems Engineer, Systems Analysis and Concepts Directorate, Space Mission Analysis Branch (E402)/Mail Stop 462, Senior Member AIAA. 


$\begin{array}{ll}\text { ISRU } & =\text { In-Situ Resource Utilization } \\ \text { LEA } & =\text { Launch/Entry/Abort } \\ \text { MACES } & =\text { Modified Advanced Crew Escape Suit } \\ \text { NEA } & =\text { Near-Earth Asteroid } \\ \text { NEOO } & =\text { Near-Earth Object Observations } \\ \text { NRE } & =\text { Non-Recurring Engineering } \\ \text { PLSS } & =\text { Portable Life Support System } \\ \text { RBC } & =\text { Robotic Boulder Capture } \\ \text { ROSA } & =\text { Roll-Out Solar Array } \\ \text { SAC } & =\text { Small Asteroid Capture } \\ S E P & =\text { Solar Electric Propulsion } \\ S L S & =\text { Space Launch System } \\ S T M D & =\text { Space Technologies Mission Directorate }\end{array}$

\section{Introduction}

$\mathrm{T}$ HIS paper discusses some of the key technologies and capabilities utilized in the Asteroid Redirect Mision (ARM) that are applicable to other applications for NASA and Human Space Exploration, thus representing potential opportunities for extensibility. First, a breakdown of the ARM is provided, along with a short description of what each mission segment entails. The capabilities used in each mission segment that are extensible to other NASA applications are then thoroughly discussed.

The ARM, as it is currently defined, consists of three segments: 1) Observation Campaign; 2) Asteroid Redirect Robotic Mission (ARRM) and 3) Asteroid Redirect Crewed Mission (ARCM). The Observation Campaign utilizes ground-based telescopes and in-space assets such as the Spitzer Telescope to identify potential asteroid targets based on a set of required characteristics. Two primary options are being studied for the Asteroid Redirect Robotic Mission. The Small Asteroid Capture (SAC) option sends the Asteroid Redirect Robotic Vehicle (ARRV) to capture an entire small asteroid (4-10m mean diameter). The Robotic Boulder Capture (RBC) option sends the ARRV to a larger asteroid, such as Itakowa or Bennu, to return a boulder from the surface of the larger asteroid. For both options, the ARRV returns the asteroid material to a Distant Retrograde Orbit (DRO) around the Moon. The Asteroid Redirect Crewed Mission utilizes the SLS and Orion spacecraft to send a crew of two astronauts to the DRO to investigate the asteroid material and return a sample to Earth. Each of these segments is described in the following sections, followed by the capabilities and technologies that are extensible to other NASA applications and future programs. Potential subsequent missions that can build upon the ARCM are discussed in section V.

\section{Observation Campaign Extensibility}

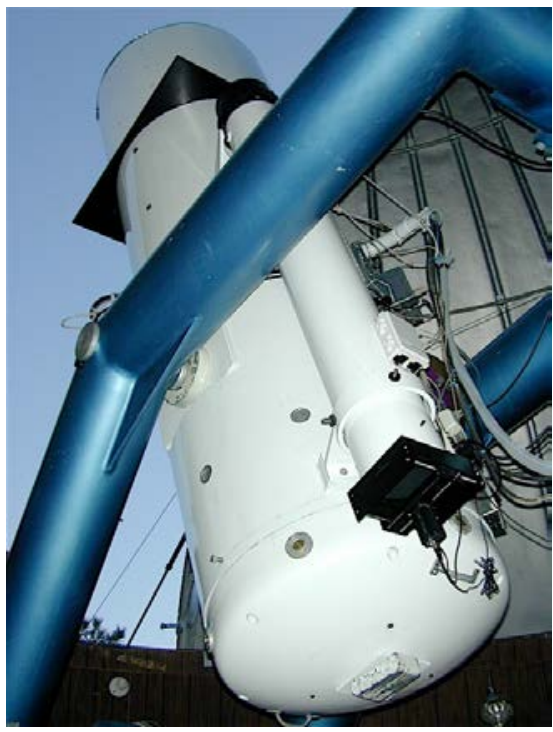

Figure 1. Catalina Sky Survey Telescope

The observation campaign for ARM leverages off of NASA's Near-Earth Object Observations (NEOO) Program, which leads the international Near-Earth Asteroid (NEA) discovery and characterization effort. Since 1998, the NEOO Program has been largely responsible for finding over $95 \%$ of the $1-\mathrm{km}$ and larger NEAs which could pose a threat to the Earth. Over the last 7 years, the program's objective has shifted to finding hazardous NEAs down to about 140 meters in size, which still could pose a significant threat. For the SAC option, in which an entire asteroid would be brought back to lunar orbit, the NEOO Program is tasked with searching for much smaller asteroids, less than $\sim 10$ meters in size and in very Earth-like orbits, so that they can be easily captured into lunar orbit. The search process is essentially the same for all asteroid sizes, and in fact a handful of potential ARM candidates had already been found with roughly the right size and suitable orbits. None of these candidates, however, had its size and mass characterized well enough to become a serious candidate for the mission, and most will be too far from Earth to be characterized before 2018. Fortunately, two of the candidates, 2009 BD and 2011 MD, passed close enough to the Spitzer Space Telescope 
(illustrated in Fig. 2) to be reasonably well characterized. It is important to note that only upper bounds could be determined for 2009 BD because it turned out to be too small to be fully characterized.

For the RBC option, in which a boulder would be removed from the surface of a 100-meter-class asteroid and brought back to lunar orbit, the surface of the asteroid must be characterized sufficiently well so that the presence of boulders can be inferred. The Itokawa asteroid has already been well characterized by the Japanese Hayabusa mission and is a suitable candidate. The Bennu asteroid should also be suitable once the OSIRIS-REx mission arrives in 2018. Asteroid 2008 EV5 is also considered a valid candidate, since it passed close enough to Earth for radar to detect boulders on its surface.

To augment the list of suitable ARM candidates for both ARM mission concepts, the observation campaign is being

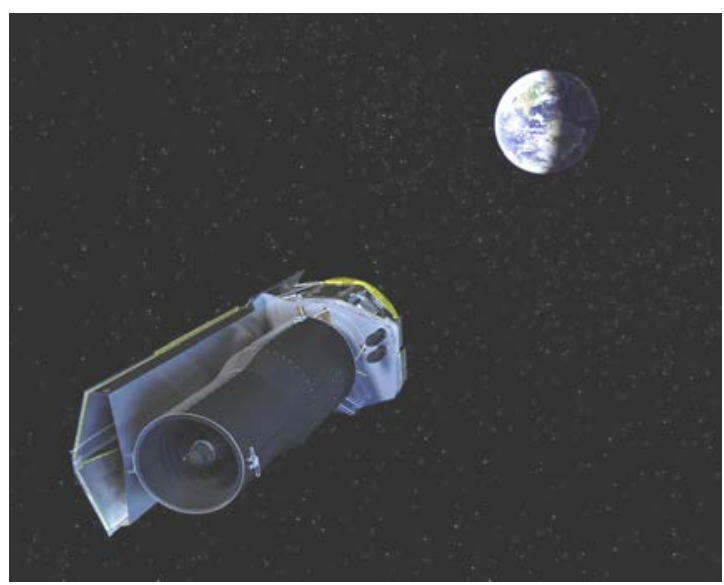

Figure 2. Spitzer Infrared Space Telescope. enhanced. New telescopes are coming online, and existing surveys are being upgraded with greater time allocations and improved cameras. Conservative projections indicate that the discovery rate of candidates for the SAC option should double to about 5 per year. The ideal time to characterize the physical properties of an ARM candidate is usually very soon after discovery, when the asteroid is still near the Earth and can be observed with ground-based radar as well as optical and infrared telescopes. Most of the new discoveries should be immediately characterizable. The discovery rate of characterizable candidates for the RBC option should also increase to a few objects per year. With the enhancements to the observation campaign, as many as a dozen additional potential candidates for both concepts should be available before the end of 2017 when a final target must be selected. It is important to note that the enhancements to the observation campaign will also increase the discovery rate of hazardous asteroids in general.

\section{Asteroid Redirect Robotic Mission Extensibility}

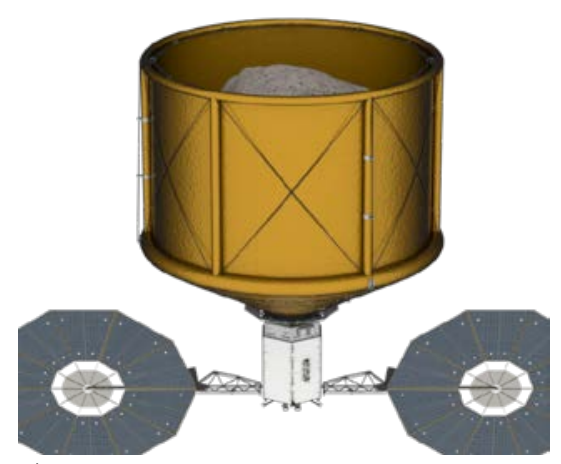

a)

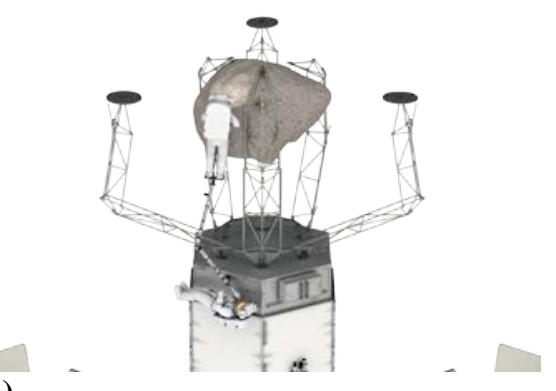

b)

Figure 3. ARRV Designs for a) Small Asteroid Capture and b) Robotic Boulder Capture options.
As previously mentioned, the Asteroid Redirect Robotic Mission (ARRM) team is currently assessing two concepts for the return of asteroid material to cis-lunar space. The primary difference between the two concepts is the size and mass of the target and the related capture mechanism. The first option, as illustrated in Fig. 3a (with a 10-meter diameter asteroid), includes the use of a large inflatable structure in the capture of a whole small asteroid up to 10 meters in diameter and up to 1000 metric tons of mass. The second option includes the use of either low or high degree of freedom (DOF) manipulators for the acquisition of a boulder up to 4 meters in diameter and up to 70 metric tons of mass from a larger parent asteroid. Figure 3b shows a low-DOF concept with a 3-meter diameter boulder. Both systems would use the same solar electric propulsion (SEP) bus in the execution of their respective missions.

Launch of either option could be on an SLS or a commercial expendable launch vehicle (ELV). Launch on an SLS would shorten the transit time departing the Earth's gravity well given its higher performance. Transit to the target NEA or larger asteroid using low power SEP would take a minimum of 2 to 3 years depending upon the orbital mechanics of the target and launcher selection. It is at the arrival of the target where the operations of the two options significantly differ.

Upon arrival at the targeted NEA, the ARRV based on the SAC option would initiate full characterization of the asteroid including size, mass, spin rate. The data would be used in determining the best approach on the final capture and control of 
the target as well as help scientists initiate planning for the future Extra-Vehicular Activities (EVAs) to collect samples. The current assumption is that the spacecraft would then be used to demonstrate ion beam deflection techniques in support of planetary defense objectives. Once these objectives are satisfied, the spacecraft would be commanded to align to the primary axis of rotation of the small NEA and match the spin rate up to $0.5 \mathrm{rpm}$. The inflatable capture systems would then be deployed and, after confirmation of good deploy, would be commanded to automatically and autonomously envelope, capture, and despin the asteroid to establish full attitude control. The spacecraft with the captured asteroid would then proceed to use the low thrust system of the SEP to alter the orbit of the NEA, redirecting it to a pre-determined DRO in cis-lunar space during its 3 to 4 year return.

In the RBC option, the ARRV arrives at a larger parent asteroid, such as Itokawa, and begins to perform characterization fly-bys in order to verify and refine parent asteroid shape, spin, and gravity models, as well as to obtain approximately cm-resolution imagery of the surface in the process of determining the potential boulder targets. Once the boulder targets have been identified, a series of dry runs which allow for higher resolution imagery and the evaluation of navigation and integrated system performance in the low gravity environment will be conducted. The concept includes up to 5 capture attempts to handle contingencies against surface and boulder anomalies. Once the target has been selected and performance confirmed, the spacecraft will be commanded to a position 50 meters above the target. At this point the spacecraft would be commanded to proceed to automatically and autonomously descend to the asteroid surface, collect the boulder, and ascend to a position safely away from the parent asteroid. With successful collection of the boulder, the spacecraft will then be commanded to an "orbit" in front of the parent asteroid's orbital path to demonstrate the effectiveness of the enhanced gravity tractor technique for planetary defense. After a period of several weeks to months, the spacecraft with the captured boulder would be commanded to return to Earth and enter a DRO in cis-lunar space similar to the first option.

The ARRM offers various technologies that are candidates for extensibility. These technologies are: solar electric propulsion, asteroid resource utilization, and planetary defense. In addition, the RBC option can be extensible to other NASA applications. The extensibility of these applications is discussed herein.

\section{A. Solar Electric Propulsion Extensibility}

Solar electric propulsion (SEP) has been used in various commercial, Department of Defense and NASA spacecraft. There are now hundreds of commercial spacecraft operating in geosynchronous orbit utilizing several forms of electric propulsion (EP). Three of the US Air Force's set of Advanced extremely high frequency (EHF) satellites launched in 2010, 2012 and 2013 have all used Aerojet's BPT-4000 thruster operating at $4.5 \mathrm{~kW}$, now the highest power Hall thruster flown in space. The first Advanced EHF satellite suffered a failure of its apogee engine but utilized a combination of reaction control propellant and the BPT-4000 system to ultimately reach the assigned geosynchronous position without affecting the overall design mission life. ${ }^{1}$

Over the past several years, there have been several investments in SEP by NASA, both in the larger solar arrays and in higher power electric propulsion systems. NASA's Space Technologies Mission Directorate (STMD) awarded two contracts in 2012 to mature technologies needed for lightweight, higher-power solar arrays. The objective of these contracts is to develop 30 to $50 \mathrm{~kW}$ arrays (beginning of life at $1 \mathrm{AU}$ ) that are also extensible to power levels of $250 \mathrm{~kW}$ or more ${ }^{2}$ and perform various ground-based tests on the demonstration units. ATK's MegaFlex is currently undergoing thermal vacuum deployment tests at NASA Glenn Research Center Plum Brook Station. The MegaFlex design is a $9.6 \mathrm{~m}(32 \mathrm{ft}$ ) diameter solar array. Deployable Space Systems (DSS) is developing their Roll-Out Solar Array (ROSA) which is about 4.5 meters wide and 14 meters long. ROSA's deployment is planned to be demonstrated in a thermal vacuum facility later this year. Both of these array systems at their demonstrated size will be capable of producing approximately $20 \mathrm{~kW}$ per wing. Beyond use in NASA missions, these array technologies offer the potential for both lighter weight and less expensive arrays for commercial spacecraft. STMD has also been conducting higher-power electric propulsion system development efforts internal to NASA at the

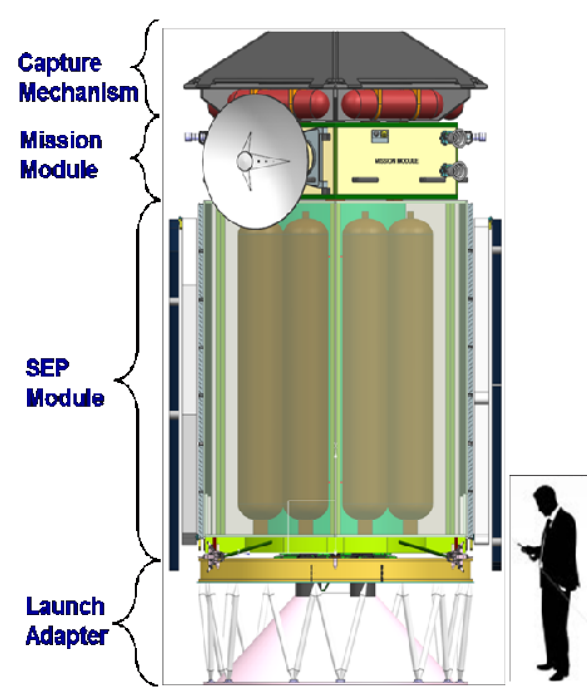

Figure 4. ARRM Reference Conceptual Configuration.

Glenn Research Center and the Jet Propulsion Laboratory. Later this year, this project will test a long-life Hall thruster system able to be operated in the $8-15 \mathrm{~kW}$ power range at 2000-3000 seconds of specific impulse. A significant advancement of these thrusters will be the removal of the primary life limiting factor via magnetic 
shielding while operating at higher powers than current electric propulsion systems. The STMD project also has additional efforts examining the accompanying power processor units and direct drive units. The STMD's investments in higher-power solar arrays and the higher-power electric propulsion system have applicability on commercial, defense, and NASA applications, both for science and supporting human exploration. One of the specific missions that these advanced systems have direct applicability to is the ARRM. ${ }^{3}$ Solar arrays which are larger, lightweight, and produce more power than those currently flying on operational satellites are needed to reach the levels required for the ARRM electric propulsion system. ${ }^{4,5}$ Multiple Hall thrusters operating simultaneously at a combined power level of approximately $40 \mathrm{~kW}$ is the ARRM current reference concept. Additionally, the current ARRM mission is designed to use 10 metric tons of xenon to reach the asteroid, capture it, and return it to a stable orbit in cislunar space. This is much more xenon propellant than the largest xenon propellant load launched to date - approximately $430 \mathrm{~kg}$ on NASA's Dawn mission. Figure 4 shows the reference conceptual configuration of the ARRM vehicle with the solar electric propulsion system below the mission module, while Fig. 5 shows the aft end of the reference configuration with the Hall thrusters and the passive NASA Docking System (NDS) to support Orion docking during the ARCM.

The significant capability of the ARRM vehicle, with 10 metric tons of xenon propellant being processed by a $40-\mathrm{kW}$ electric propulsion system, makes it attractive for use in other human exploration missions. The SEP system on ARRM could be utilized in an evolutionary nature on a range of missions: from other longer-duration near-Earth missions in cislunar space with similar capability as for ARRM, to more demanding missions such as cargo delivery for human Mars missions. ${ }^{6}$ Mars cargo missions will require higher power for SEP systems than that for ARRM, but the solar array systems being demonstrated under the STMD effort will show their extensibility to $125 \mathrm{~kW}$ per wing via modularity and

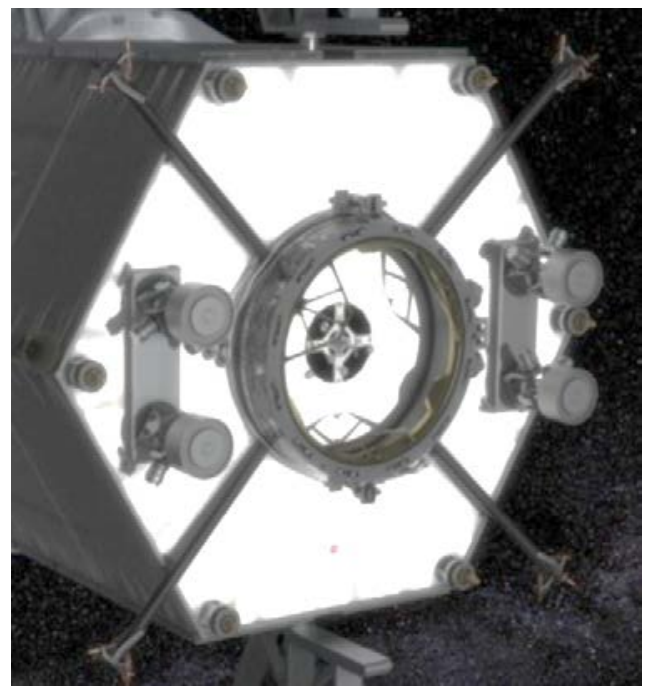

Figure 5. Aft of ARRV Reference Concept showing Hall Thrusters, RCS pods and passive NDS. scalability. When combined with either a greater number of the ARRM electric propulsion system strings or with a set of higher power EP strings, it could easily reach the capability required for timely transportation of large cargo elements to Mars. The SEP on the ARRM could be the basis of this extensibility in several ways: 1) it could be scarred to accept a set of planned changes as it is used on more demanding missions; 2) it could be used in modular fashion where multiple SEP elements could be joined together, making building blocks of 80 - or 120-kW worth of SEP; or 3) a complete block upgrade approach could be used where the subsystems other than those supporting SEP are standardized and larger, higher-power SEP subsystems would be adapted as the more demanding mission is undertaken.

All of the current STMD technology maturation efforts in both solar array and electric propulsion systems will be applicable to both ARRM and more-demanding human exploration missions. Through operating the set of systems needed for the subsequent exploration missions, ARRM will demonstrate the next step of advanced in-space propulsion supporting the needs of NASA, which could also be used in commercial spacecraft.

\section{B. Asteroid Resource Utilization Extensibility}

One of the critical resources for future human space exploration is water. Water can be used for a variety of purposes, including propellants, radiation shielding, thermal control, human consumption, as well as a variety of non-potable applications (cleaning, plant growth, etc.). Carbonaceous (C-type) asteroids may contain up to 20\% water by mass in the form of hydrated minerals. C-type asteroids may also have similar mass percentage of other extractable volatiles. In addition to water and other volatiles, a number of other resources are available from asteroidal materials. These include oxygen from mineral oxides, metals (e.g., iron, nickel, and platinum group metals), and bulk materials that can be used for radiation shielding and the construction of various structures.

Determining the composition of the target NEA is critical, and although it is technically possible to remotely characterize the composition of a small NEA, targeting a large, well-characterized NEA significantly increases the potential for returning water/volatile-rich material from a carbonaceous NEA. C-type asteroids also possess low compressive strength that simplifies cutting, crushing, and processing, which could be a key target characteristic for practical resource extraction and recovery. Returning the right type of asteroidal material to cislunar space would allow in-situ resource utilization (ISRU) demonstrations to be conducted on the material to determine how 
successfully the bulk asteroidal material can be converted into useful products. This would be beneficial for future human exploration of the solar system and could be critical in advancing commercial efforts to mine asteroids. In addition to resource extraction, the analysis and understanding of water and water-bearing minerals from asteroids is extremely important to our understanding of solar system formation and the origins of life on Earth. Figure 6 depicts a concept for a large-scale future propellant processing facility attached to a large asteroid.

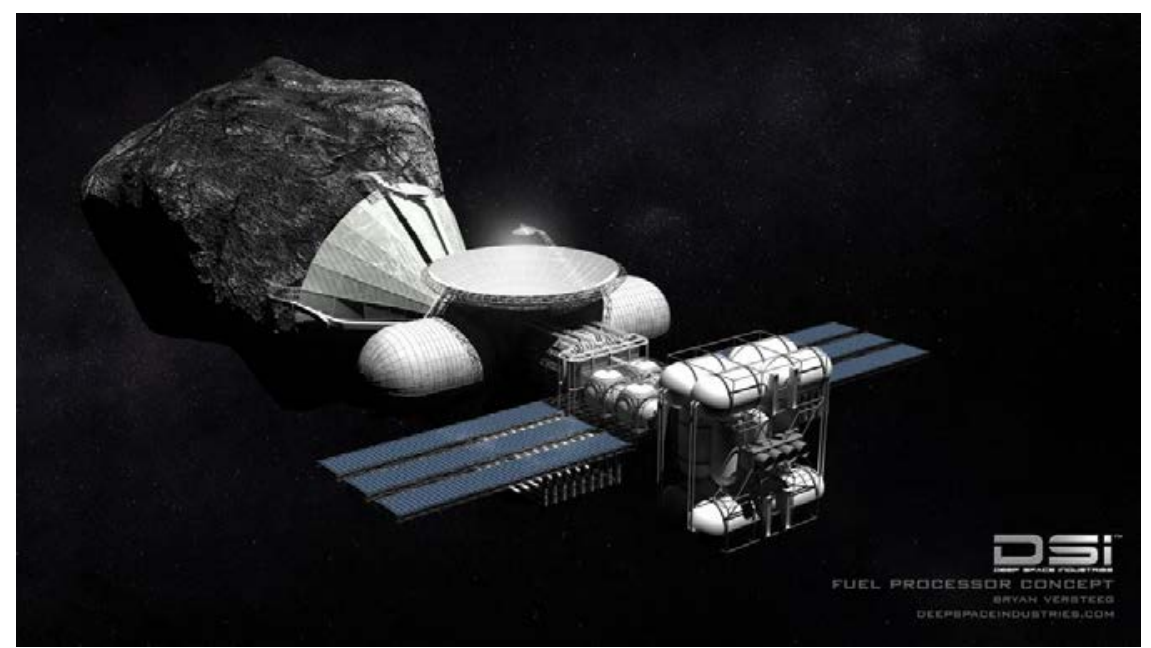

Figure 6. Asteroid Propellant Processing Concept (Image Credit: Bryan Versteeg / Deep Space Industries).

\section{Robotic Boulder Capture Extensibility}

The use of robotic arms to interact with a NEA and capture a boulder from its surface has many possible applications to other NASA missions and uses by commercial companies and international partners. The extensible activities include satellite servicing and refueling, providing surface contact and manipulation for a future crewed mission to NEAs or the Martian moons, and providing critical experience operating around and on a low-gravity planetary body. Boulder collection operations currently assumed in the RBC option include planned routines with fault tolerance for boulder acquisition and asteroid contact, which will be needed in support of future autonomous operations in deep space. In addition, endeffector/grippers such as the microspine technology being developed for the ARRM RBC option can be used for future robotic exploration of lunar lava tubes, vertical cliffs on the Martian surface, and the exploration of bodies like NEAs, Phobos, Deimos, and main belt asteroids like Ceres.

Two capture systems approaches have been the focus of analysis for the RBC option. The first approach is a three degree

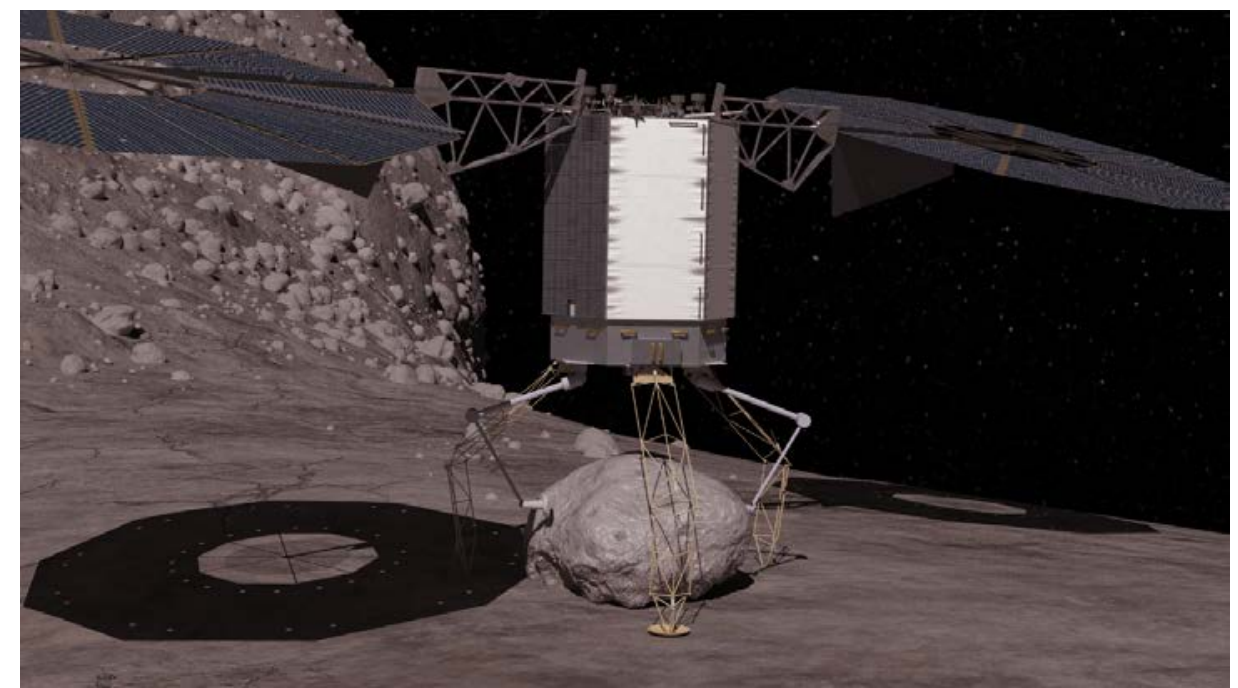

Figure 7. Hybrid Capture System Concept for the ARRM RBC Option (Image Credit: NASA/AMA, Inc.).

of freedom (3-DOF) spaceframe capture system that employs a simple, repetitive design with a minimum number of unique machined parts and no new technology that can be used to capture a boulder and mechanically push off from the NEA's surface. The second approach uses seven degree of freedom (7-DOF) robotic arms with microspine grippers to provide additional dexterity and operational flexibility. The 7-DOF arms have significant heritage and 
can leverage significant prior non-recurring engineering (NRE) investments. Three 3-DOF Spaceframe contact arms and two 7-DOF arms are depicted in the hybrid capture system depicted in Fig. 7. The hybrid capture system optimizes functionality and maximizes extensibility of concept. Figure 8 provides a depiction of four spaceframe capture arms integrated with a notional Phobos Habitat on the surface of Phobos, one of Mars' moons. In this potential scenario, the ARRV SEP bus could be utilized to pre-position Mars Moon mission assets in support of a crewed mission to Phobos. Such Mars missions would require continuing to evolve the solar and EP systems to perform in the 100-250kW range.

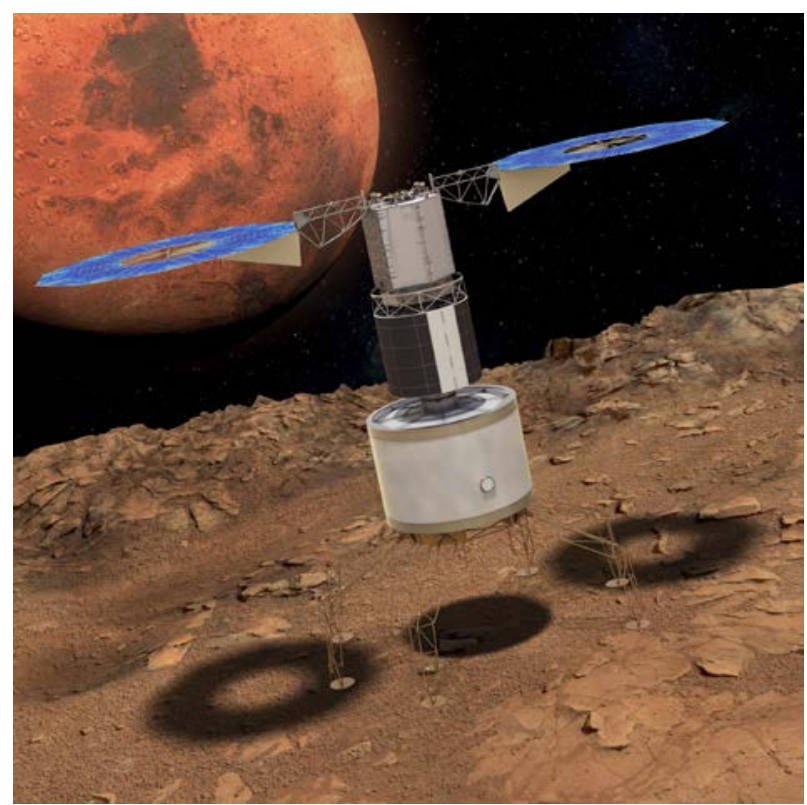

Figure 8. Spaceframe Capture Arms integrated with a Habitat on the Phobos Surface (Image Credit: NASA/AMA, Inc.).

\section{Planetary Defense Extensibility}

Although planetary defense demonstrations can be performed for the two ARRM options, the techniques, relevance, and extensibility is significantly improved by conducting a mission to a hazardous-size NEA. The size of NEA targeted by the SAC option is below the threshold that can typically survive entry through the Earth's atmosphere, and therefore is not of a hazardous-size. The RBC option provides critical planetary defense experience by operating at a large NEA, both through sustained orbital operations and extended surface interactions. Planetary defense demonstrations that can be performed while at the target NEA include the Enhanced Gravity Tractor (EGT), Ion Beam Deflection (IBD), and the option to test a kinetic impact approach.

The EGT technique, which is the focus of the RBC option, utilizes the acquired boulder to augment the mass of the spacecraft. This greatly increases the technique's effectiveness, and the current RBC option mission timeline reserves 180 days for EGT operations with as little as 60 days required for a measurable deflection of a multihundred meter diameter NEA. The ARV orbits the NEA in a halo orbit that maintains a safe, constant distance from

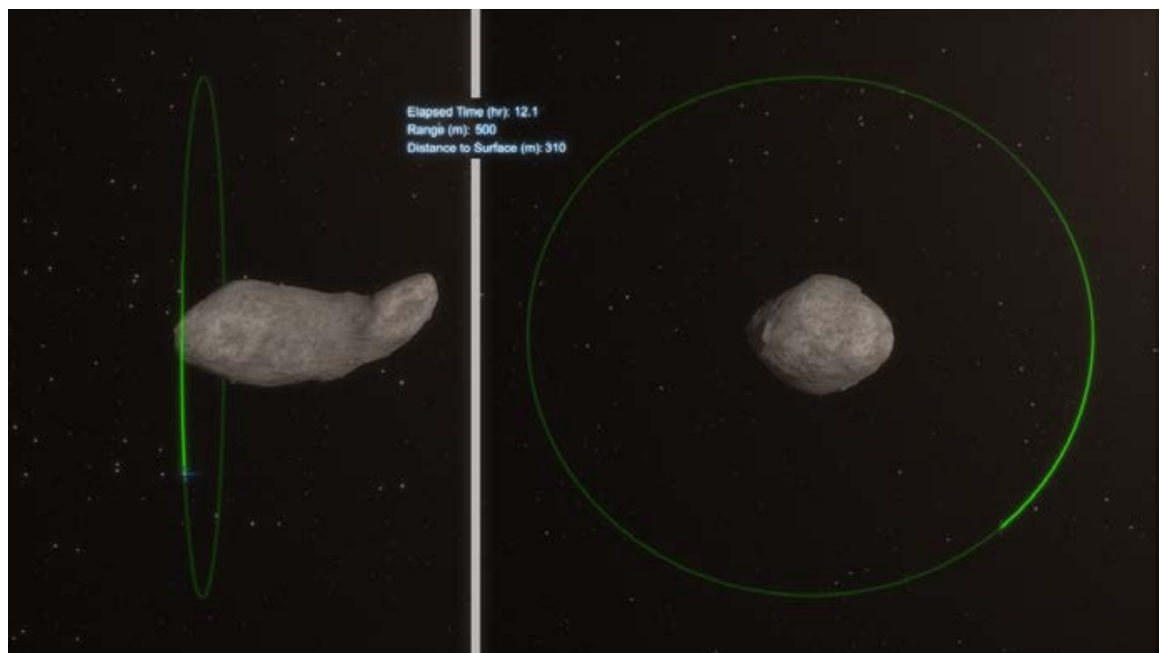

Figure 9. Demonstration of the Enhanced Gravity Tractor on a Large NEA (Image Credit: NASA/AMA, Inc.).
NEA as depicted in Fig. 9. The ARV utilizes the continuous SEP thrusting to counteract the NEA gravitational force and solar radiation pressure, and can use chemical impulses or just the SEP system to maintain the halo orbit and account for navigation and control errors as well as unmodeled perturbations.

Given sufficient warning time, the EGT approach is applicable to diverting NEAs from Earth-impacting trajectories. For an actual planetary defense mission to avoid an Earth impact, the 
acquired boulder would not need to be returned to the Earth-Moon system and a much more massive boulder (or boulders) could be retrieved and provide significantly more mass augmentation that could be achieved during the ARRM. Additionally, multiple ARRVs could be dispatched to the impacting NEA and work in a concerted manner to provide the necessary velocity change. Diverting a confirmed impactor approximately $300 \mathrm{~m}$ in diameter could require operation times of five or more years with a single ARRV and a captured boulder with a mass of 100 metric tons. Without many years of warning time, an impulsive technique such as a kinetic impactor may be required to provide the necessary velocity change. This technique was demonstrated by the Deep Impact mission when a 370-kg impactor was successfully targeted at Comet 9P/Tempel on July 4, 2005 (see Fig. 10). Comet 9P/Tempel is a small body that is approximately $8 \mathrm{~km} \mathrm{x} 5 \mathrm{~km}$ in size. Impacting a smaller NEA ( 300-1000 m) with a similar mass impactor will provide significantly more change in velocity, but will also require better targeting accuracy. The targeting accuracy would be improved by having the ARV near the target, but observing at a safe distance. After the boulder collection operations and other

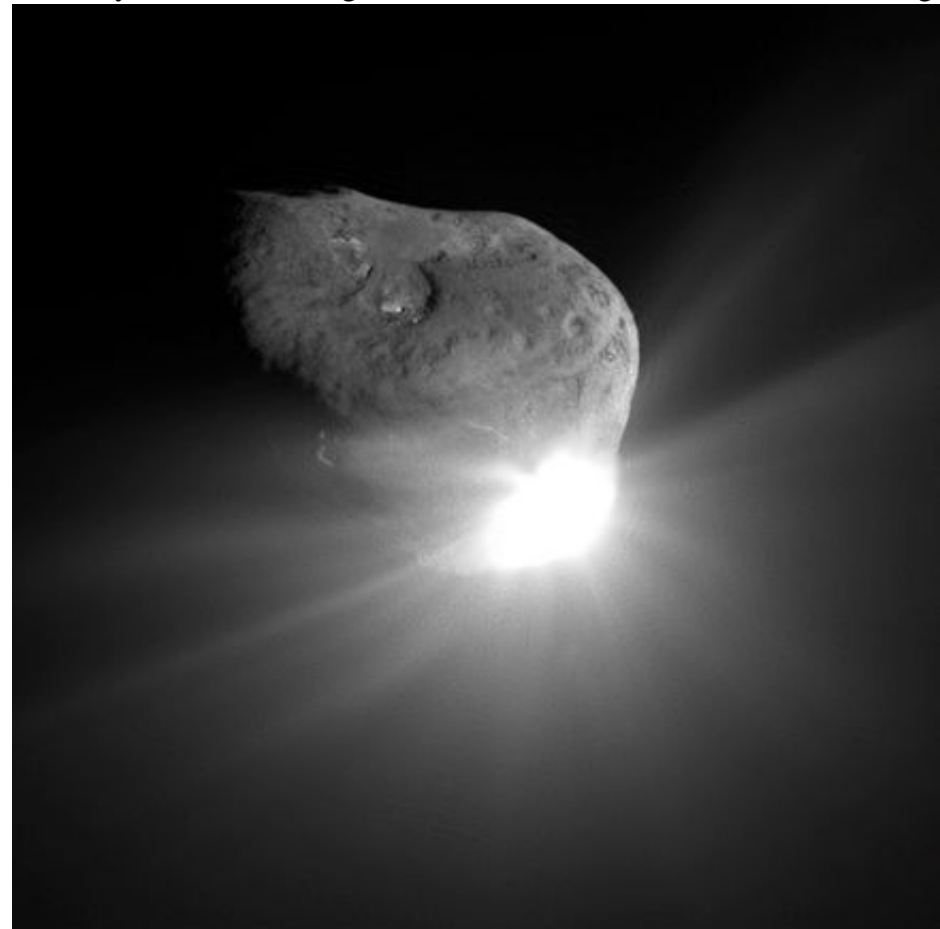

Figure 10. Kinetic Impact on Comet 9P/Tempel by the Deep Impact Mission (Image Credit: NASA).

planetary defense demonstrations are complete, a kinetic impact demonstration could be conducted for the RBC option and the effectiveness of the technique compared to the other non-impulsive techniques performed at the target NEA. A kinetic impact demonstration for the SAC options is not viable for the obvious reason the kinetically impacting the targeted NEA is not compatible with subsequently capturing the small NEA.

\section{Asteroid Redirect Crewed Mission}

Once the ARRV with the captured asteroid or asteroid boulder is in the DRO in cis-lunar space, a two-man crew in the Orion spacecraft launched on the SLS will execute a mission to return up to $100 \mathrm{~kg}$ of samples from the returned target. In addition to the acquisition and return of these samples, the execution of this mission will demonstrate the capabilities of SLS and Orion to support translunar missions, the rendezvous and docking system of Orion with the robotic vehicle, and deep space EVA and tools, all of which represent potential candidates for extensibility for other NASA applications.

In order to execute the mission, both the Orion Spacecraft and ARV are augmented in the form of mission kits. The Orion is designed to support four crew on 21-day missions beyond low Earth orbit and does not support EVA. Reducing the crew size from four to two provides additional internal stowage and mass capability. This recovered volume allows for the addition of ARCM mission kits which will extend the capability of the Orion to support the ARCM flight. The fundamental design guideline for the kits is to provide the necessary functions self-contained within the kits with minimal changes to the baseline Orion configuration and ground support equipment. The additional functionality required on the Orion spacecraft includes an active NASA Docking System (NDS), the automated rendezvous and docking navigation system, the Modified Advanced Crew Escape Suit (MACES) pressure suit system with an exploration class Portable Life Support System (PLSS), EVA tools and sample containers, and a repressurization system for the Orion CM.

The ARRV will require the addition of up to three small retro-reflector assemblies and an S-Band system to support proximity operations with the Orion vehicle. A passive docking mechanism will be added to the aft end of the ARRV, opposite the asteroid capture mechanism, to facilitate docking with Orion. The docking mechanism will contain a docking target for Orion relative navigation sensors and crew situational awareness. 


\section{A. Common Automated Rendezvous Sensors}

NASA needs automated rendezvous and docking/capture (AR\&D) sensors for both the robotic and crewed segments of the ARM. NASA recently conducted a commonality assessment of the concept of operations for both the Asteroid Redirect Robotic Vehicle (ARRV) concept and the crewed mission segment concept using the Orion crew vehicle. The commonality assessment also considered several future exploration and science missions requiring an AR\&D capability such as asteroid sample return, satellite servicing, as well as planetary entry, descent, and landing.

This assessment determined that a common sensor suite consisting of one or more visible wavelength cameras, a three-dimensional LIDAR along with a long-wavelength infrared camera for robustness and situational awareness could be used on each mission to eliminate the cost of multiple sensor developments and qualifications. By choosing sensor parameters at build time instead of at design time and eliminating multiple flight hardware qual cycles, a specific mission can design overlapping bearing, range, relative attitude, and position measurement availability to suit their mission requirements with minimal nonrecurring engineering costs. The resulting common sensor specification provides the union of all performance requirements for each mission and represents an improvement over the current systems used for AR\&D today. Figure 11 summarizes the concept of operations for each of the asteroid mission concepts and how that mission will apply sensors from the common suite.

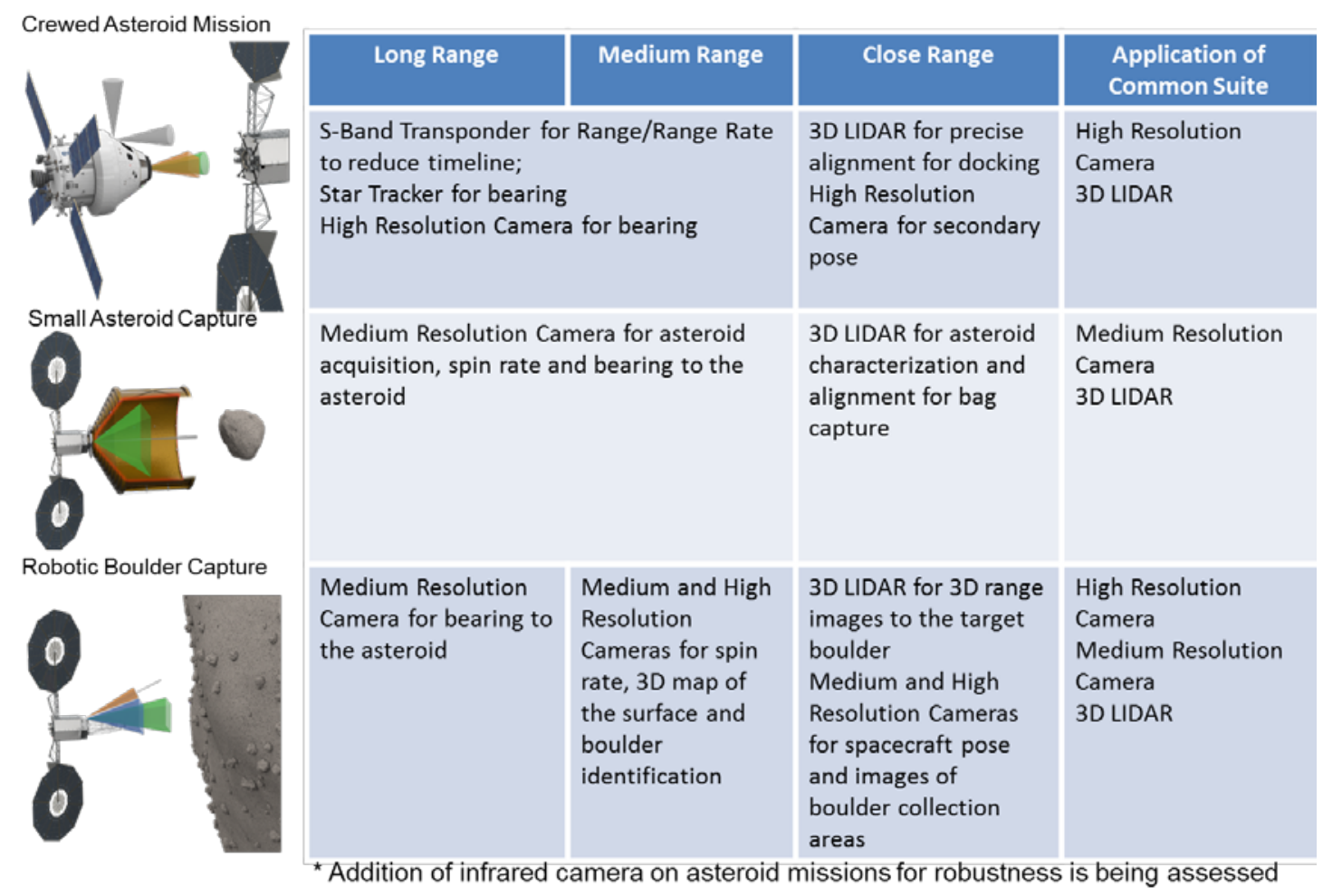

Figure 11. AR\&D Concept of Operations for Asteroid Mission Concepts

NASA acknowledges that as technology improves, changes, and matures, the common sensor suite will need to change and adapt. NASA is looking into how to incrementally improve this sensor suite as technology improves and advances. Examples include advances in the regime of noise radar, computing, and other technologies that could enable new approaches to AR\&D. New technologies could result in savings on size, weight, power, and complexity. NASA would like to establish incremental growth in AR\&D/C capability including the ability to operate in parallel to other solutions to demonstrate the technology prior to integrating it into the primary solution set.

\section{B. Extravehicular Activity (EVA)}

There are many aspects of the EVA segment of the crewed asteroid mission that are extensible to other missions. The technologies, techniques and operations concepts being developed could extend to manned missions to the Earth's moon, other asteroids, the moons of Mars, or even to the Martian surface. 
The most immediate benefit will be providing contingency EVA capability to all manned Orion missions. The asteroid mission presents an opportunity to build up all of the capabilities needed for conducting capsule-based EVAs from Orion. Orion's cabin has the capability to go to vacuum, but it does not have dedicated EVA communications capability. The Service Module has one contingency repress, but it does not have enough air for multiple represses. There are currently tasks being conducted to define the operations concept for EVAs from the Orion cabin. Those ops cons will help identify areas that need more development.

The Launch/Entry/Abort (LEA) suit already manifested to be used by the crew

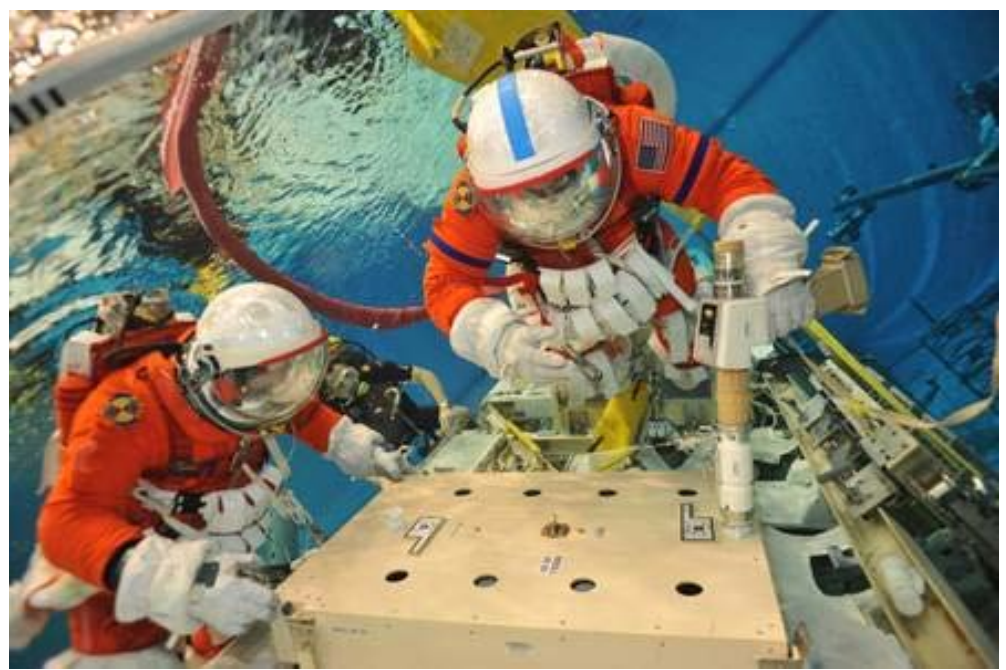

Figure 12. Mobility Enhancements Testing of MACES in the NBL.

for emergency survival situations must be utilized to successfully conduct an EVA from the Orion cabin without

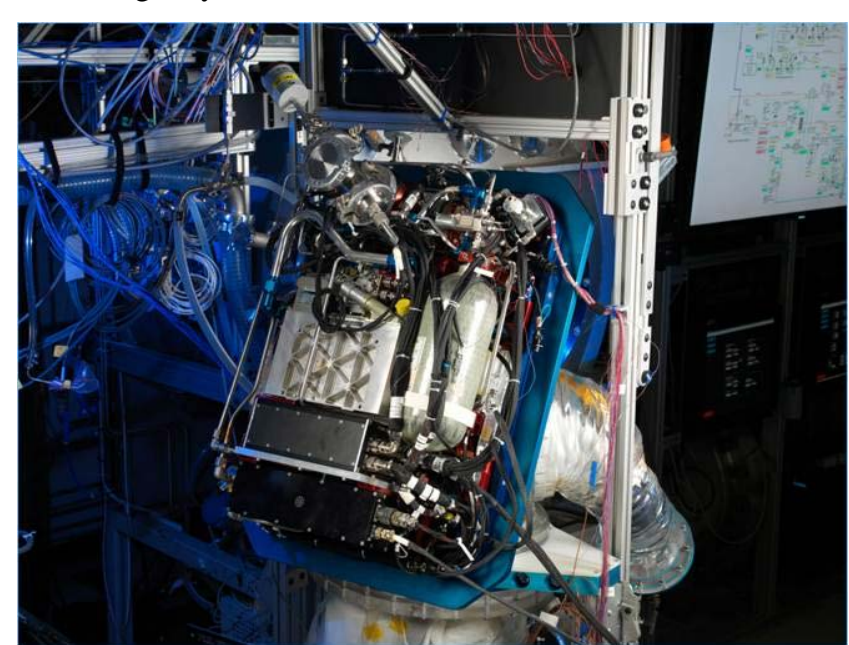

Figure 13. PLSS 2.0 Test Unit on the Space Suit Assembly bringing a dedicated EVA suit. There is precedent for using this technique. The Modified Advanced Crew Escape Suit (MACES) that now serves the LEA function is a direct descendent of the Gemini flight suits that conducted the first American EVAs in the 1960s. The lessons learned from those EVAs show that improvement is needed in the mobility of pressure suits to increase the probability of success for the EVA objectives. By adding mobility enhancements to the MACES, the suit is a more capable EVA suit while still maintaining the core function of protecting the crew. Work is being done now in the Neutral Buoyancy Lab (NBL) to identify the mobility enhancements that are needed as well as how to implement them, as illustrated in Fig. 12.

For contingency Orion EVA, the life support will be supplied to the suit by an umbilical through

the Orion ECLS system. However, the umbilical is not long enough for the asteroid mission, so life support will be supplied by a Portable Life Support System (PLSS). For the ARCM, the Exploration PLSS being developed by the Advanced EVA Development AES project is currently being considered (see Fig. 13). It is being designed primarily for use on the Z-series exploration pressure garment that is being designed for use for terrestrial missions, but there is a desire for the Exploration PLSS to be suit-agnostic; this means that as long as the interfaces are the same, the PLSS can supply the necessary services to any suit. The asteroid mission gives an opportunity to interface the PLSS to the MACES and develop the PLSS to work with the MACES. The technologies that are being developed for this PLSS are extensible to exploration missions. Carbon dioxide will be removed using a Rapid Cycle Amine (RCA) swing bed which does not need the logistics support that $\mathrm{LiOH}$ cans and other $\mathrm{CO} 2$ removal techniques need. The thermal conditioning will be provided using a Suit Water Membrane Evaporator (SWME), a technology that does not need the same level of water quality that a sublimator does. This PLSS combined with the $\mathrm{Z}$ suit will be the EVA suit of the next 30 years and will accommodate all the design reference missions including microgravity asteroids, lunar surface and Martian

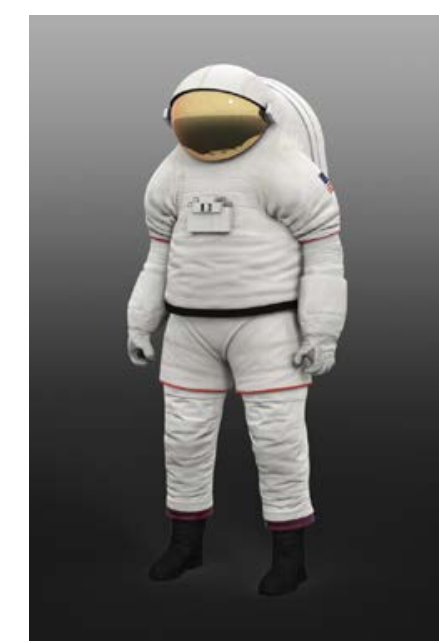

Figure 14. Z2 Suit with PLSS 
surface. Figure 14 shows a conceptual view of the Z2 suit with a PLSS.

The tools and techniques being developed for the asteroid mission are also extensible to future missions. The geologic tools being developed for the asteroid mission are extensible to any microgravity EVA including the moons of Mars. The worksite stability techniques used in ARCM will also be applicable to any other microgravity geologic mission.

\section{Sample Collection}

The EVAs conducted during the crewed mission require development of techniques and tools that are appropriate for conducting sampling operations on small airless bodies under low-gravity conditions. Previous EVA sampling experience has been limited to the Moon, which has a much different composition and surface environment, and so it may not be applicable to future small body destinations. However, some of the knowledge gained from the Apollo program, combined with the experience of previous robotic sample return missions to small bodies (e.g., Hayabusa, Stardust, etc.), can be leveraged to help refine and develop the tools and techniques required to successfully accomplish sampling of asteroidal material for the ARCM. Considerations of the microgravity environment, the types of asteroidal materials, the particle size frequency distribution, and surface geotechnical properties are just some of the aspects that need to be taken into account when developing the tools and techniques for small-body sample collection.

One of the major activities to be conducted during any crewed segment at a natural object is to collect multiple and diverse samples. Ideally, the samples collected would represent pristine materials that would not have been altered or contaminated, either through mechanical interactions (i.e., robotic or human) or via spacecraft engine plume impingement. The crew would collect macroscopic samples during EVAs from various locations of the sampled body per guidance from the ground-based science team. These activities would enable sample collection to be obtained in geological context and ensure that representative samples from the surface and/or interior can be properly maintained during the return journey for subsequent detailed laboratory analyses on Earth. For example, carbonaceous asteroid materials may have organic species or volatiles that are best preserved and stored in ambient conditions (i.e., sealed under cold temperatures). In addition, samples obtained from the fragile outer most surface will help evaluate space weathering/alteration effects of the materials' exposure to a deep space environment, and therefore require specific collection considerations and protocols. Samples collected utilizing techniques and tools developed for the ARM enhance the overall science return of the mission and will lead to a better understanding of the formation and evolution of small airless bodies.

It should also be noted that the protocols and sampling tools developed for the ARM will be directly extensible to future human missions sent to inner Solar System destinations such as other near-Earth asteroids and the moons of Mars. Samples obtained from Phobos would be of particular interest since Phobos may be similar in composition to volatile- and organic-rich carbonaceous asteroids. Such components are not only relevant for science, but also for future resource utilization considerations. Thus, lessons learned from the ARM can be applied to EVA planning for sample site assessment, documentation, sampling operations, and storage/containment activities on Phobos. Since it is possible that Phobos may have Martian material on its surface, the refined sample collection methods developed for the ARM can also be applied to the development of future planetary protection protocols.

\section{Automated Docking}

This study assumes the docking mechanism is derived from an International Docking System Standard (IDSS)compliant, passive NASA Docking System (NDS). As currently defined, the NDS contains connectors for transmission of power and data through the mechanism. Neither the hardline power nor data function is required for the ARCM, but will provide a significant resource for extensibility in the future. Figure 15 shows details of the IDSS-compliant docking interface.

Use of the NDS on the ARCM enables the use of Orion RCS for integrated attitude control while docked to the ARRV, making it possible to modify the attitude between the baseline docked attitude and the EVA Ops attitude. The NDS was also found to support subsequent missions to the DRO for asteroid utilization, especially if longerduration crewed missions are desired. The use of the NDS as the standard docking system for all spacecraft will allow for a Node-like module to be brought to the DRO, providing additional pressurized volume as well as docking ports that can be used for logistics modules, necessary to bring the required additional EVA hardware and tools as well as crew provisions and consumables.

The ability to deliver different assets in separate flights and integrate them later during the mission using the IDSS-compliant NDS would enable missions to the Mars vicinity such as Phobos and Deimos, as the mass and volume required to sustain crew for such long periods (greater than 600 days) is impossible to be delivered using existing launch vehicles as well as those currently under development. 


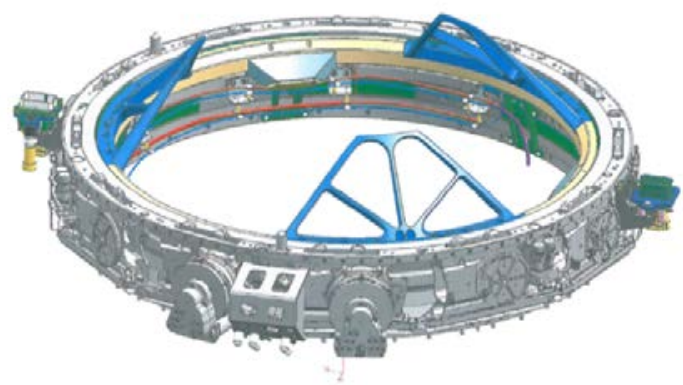

a)

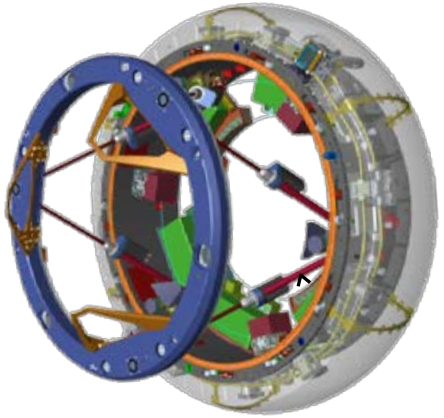

b)

Figure 15. IDSS-Compliant NASA Docking System; a) passive half on ARRV, and b) active half on Orion

\section{Extensibility to Missions Post-ARCM}

\section{A. Extended Crewed Missions to Asteroid in DRO}

Many subsequent mission scenarios following the first crewed mission are being assessed. One of the potential mission scenarios investigated after the initial crewed mission to visit the asteroid in the DRO represents longer crewed missions to the DRO with larger crew sizes to further study the asteroid, which would require additional habitable space. Longer-duration crewed missions would allow testing of in-situ resource utilization techniques as well as serve as a testbed for crew operations and systems required to sustain life for longer missions, representative of deep-space scenarios. Figure 16 shows a notional example of a long-duration architecture for asteroid utilization. In this concept, a module that provides pressurized and a number of ports to support docking of the crew transport vehicle (e.g., Orion) as well as other vehicles such as logistics modules is attached to the ARRV. The habitat also includes an EVA hatch to facilitate further exploration of the asteroid.

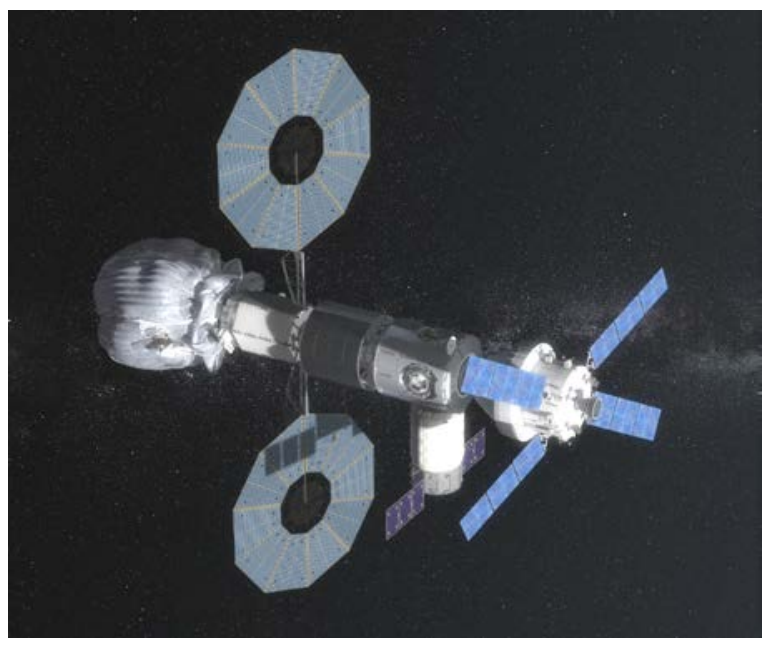

Figure 16. Notional Example of Extensibility of Asteroid Mission.

\section{B. Mars Phobos Mission}

Human exploration of the moons of Mars, Phobos and Deimos, have long been studied as a potential mission to the Martian system prior to actual landing on the surface of Mars. Such an approach would allow for development and use of key elements of the transportation system (launch vehicles, in-space transportation, deep-space habitation, and crew entry), prior to subsequent landed missions. These missions would exercise the round trip capabilities necessary to transport the crew from Earth to Mars orbit and back. In addition, while at Mars the galactic cosmic radiation exposure to the crew could be reduced if the exploration architecture can be constructed where the crew spends the majority of the time while in Mars orbit on the surface of either or both moons. While at Phobos, the crew could conduct vital exploration of the moon while also supporting teleoperation of assets on the surface of Mars for future landed missions, or perhaps even aid in return of robotically-gathered samples from the surface of Mars.

Previous studies which have explored the use of SEP in support of human missions to Mars have demonstrated that very high power, approaching one Mega-Watt, would be required. ${ }^{7,8}$ However, this high power requirement is the result of the architectural construct which uses a complete round-trip habitation system to transport the crew from Earth orbit to Mars orbit and back. This architectural assumption drives very high power demands for the SEP 
vehicle, which in turn would require yet another technological capability step beyond the ARM. Other architectures are currently being assessed which could significantly reduce the SEP power required, leading to a more directly ARM extensible system. This alternative architecture construct relies upon the "Split/Sprint" mission strategy.

Split/Sprint mission strategies have long been studied by Mars architects as a powerful tool in helping reduce the total mission mass, which for SEP concepts, can in turn reduce required power level. With the Split/Sprint approach, mission assets are sent ahead of the crew on minimum energy transfers which allows significant mass reduction. ${ }^{9,10}$ Typical pre-deployed assets include landers and Mars exploration systems and, in aggressive cases, even the stages

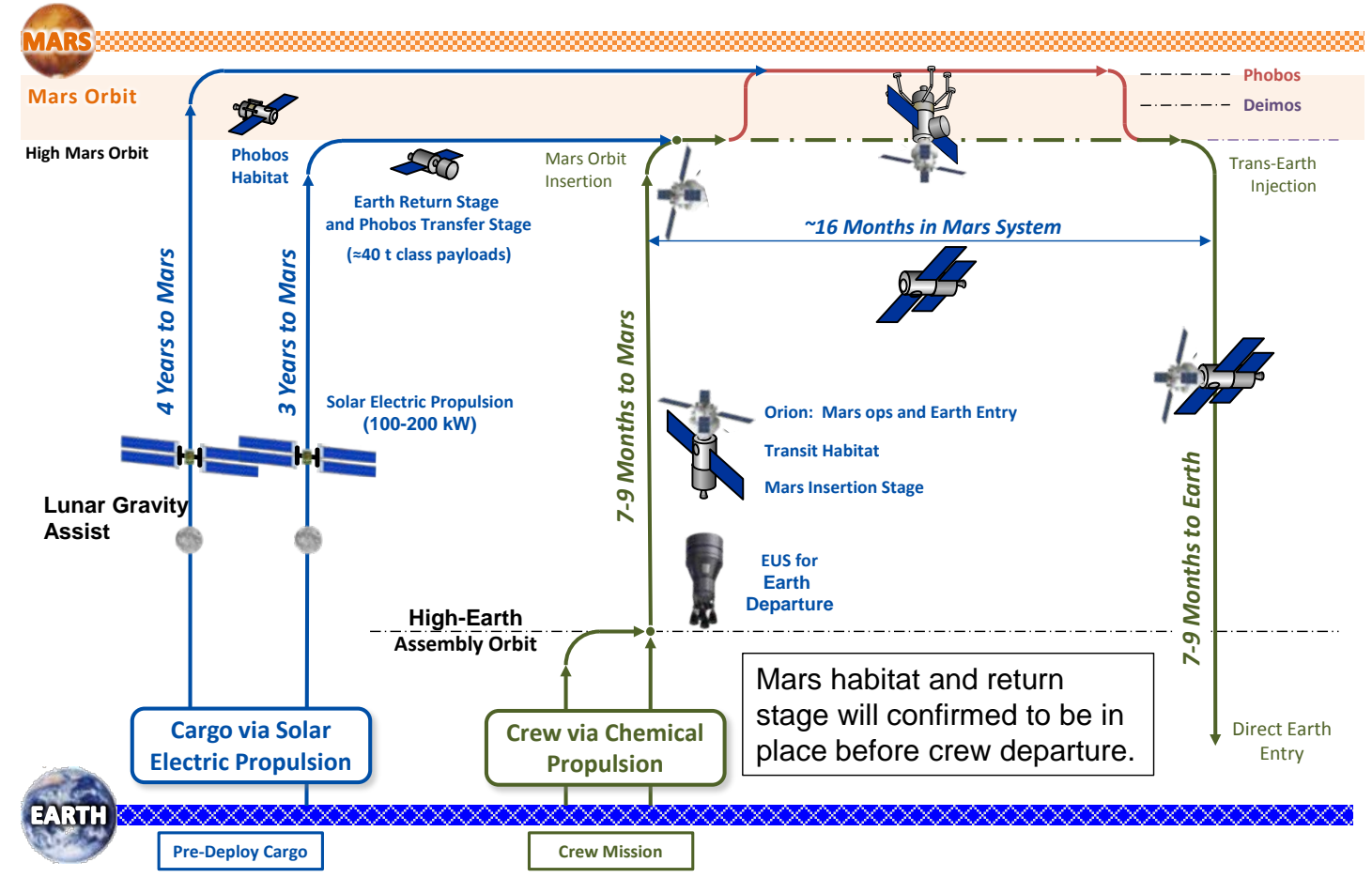

Figure 17. Notional Solar Electric Propulsion - Chemical Split/Sprint Architecture.

or propellant the crew would use to return back to Earth. Previous studies discounted the pre-deployment of return stages as being too aggressive, ${ }^{11}$ but this type of strategy is ideal for lower-power SEP/chemical architectures as shown in Fig. 17. As can be seen from this figure, SEP vehicles are used to send cargo elements out to Mars orbit ahead of the crew. Since lower power SEP systems would result in unacceptably long mission times, high-thrust chemical propulsion would be used for the crew segments of the mission. The pre-deployed assets would include the Phobos habitat and exploration systems, along with the propulsion stage that the crew would use to return to Earth. The crew would depart from Earth only after all of the pre-deployed assets have arrived in Mars orbit and are operating as expected. Pre-deploying all of these mission assets allows for a much smaller outbound crew vehicle. Since the crew is on a one-way mission to Mars, they

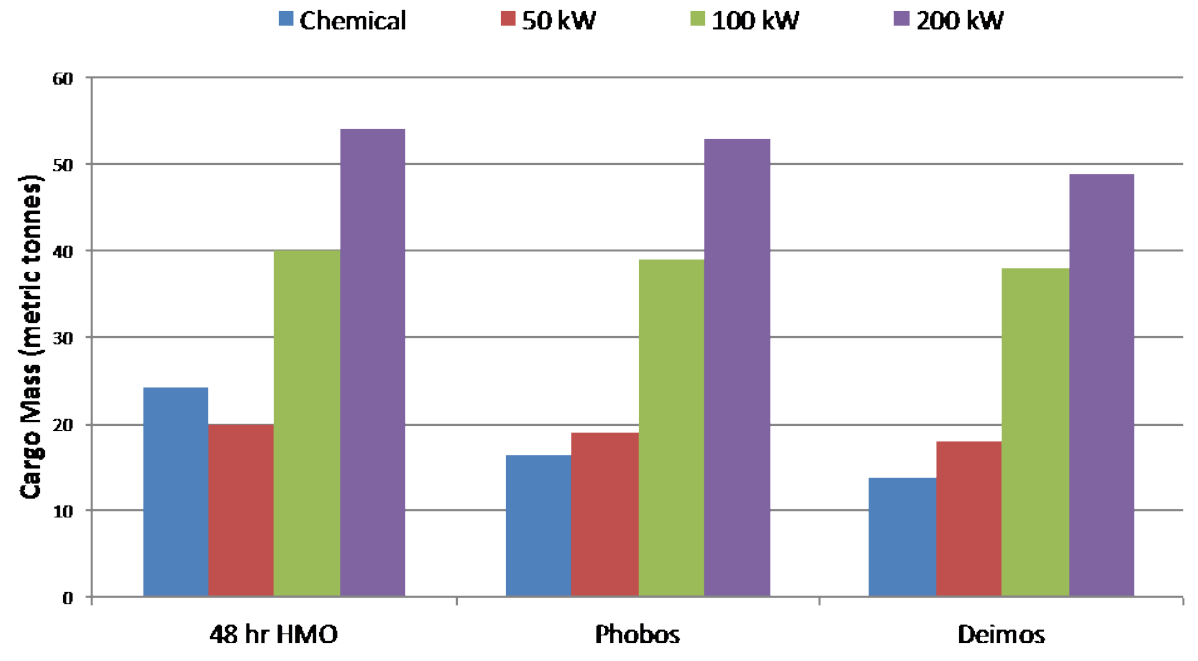

Figure 18. SEP Cargo Vehicle Performance to Different Mars Orbital Locations. 
would be required to rendezvous with the pre-deployed propulsion stage in order to return back to Earth. With this type of SEP Split/Sprint architecture the masses of the pre-deployed elements (habitat and return stage) are approximately $40 \mathrm{t}$.

SEP performance estimates, as shown in Fig. 18, indicate that power levels on the order of 100-200 kW may be sufficient to serve as the cargo leg of this type of architecture. Further assessments are still required to determine how this type of strategy can feed forward to future landed missions which require the pre-deployment of even heavier cargo elements.

\section{Lunar Mission}

Although NASA's focus is on evaluating strategies for the ARM initiative and exploration of the Mars system, the relationships of these strategies to possible lunar surface missions are also being considered in the context of the larger international approach towards human space exploration by multiple space agencies, as reflected in the Global Exploration Roadmap. ${ }^{12}$ In this regard, NASA is evaluating the potential to leverage the operational experience and extend the application of systems required for the ARM/ARCM towards an internationally coordinated effort which includes the exploration of the lunar surface.

Figure 19 depicts one of many options which could be pursued to enable human exploration of the lunar surface.

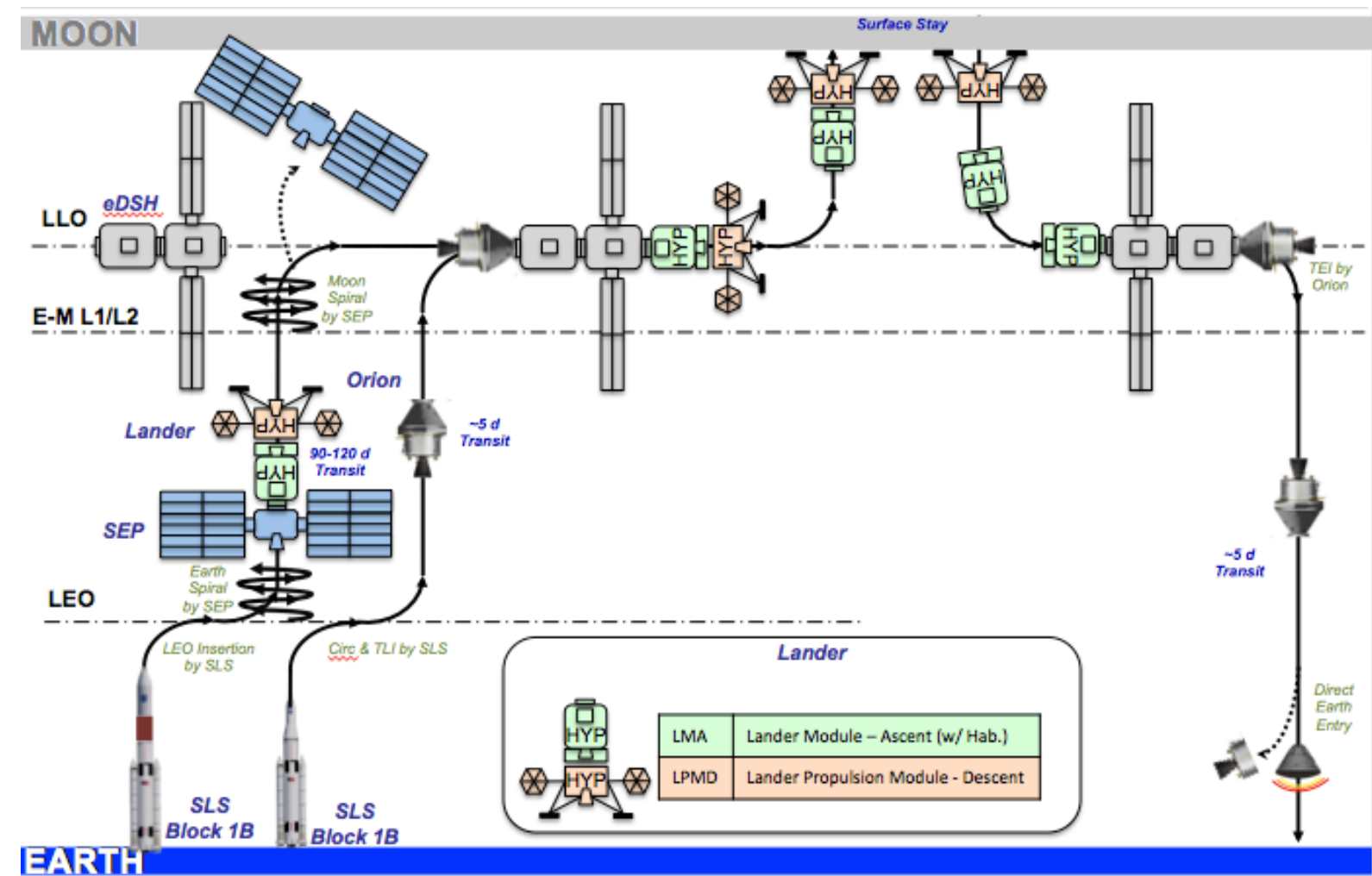

\section{Figure 19. Human Lunar Surface Mission Architecture Utilizing SEP.}

In this mission depiction, lunar surface access is enabled by a staging architecture in which an evolvable Deep Space Habitat (eDSH) is utilized to aggregate vehicle elements and crew members in a multiple launch scenario. In this scenario, the human-tended eDSH resides in lunar vicinity, where the lunar lander and potential payloads are delivered several months ahead of the crew by means of the SLS and a SEP bus/space craft via a long duration spiral from earth orbit. The SEP bus could be a production copy or a very similar version of the one used in the ARM/ARCM. Utilizing the highly efficient propulsion system to deliver uncrewed assets to lunar vicinity when time of flight is not a driving factor, allows for additional payload or mission capabilities in terms of enhanced systems where precious mass can be allocated to achieve high priority mission objectives.

\section{Capability Driven Framework}

The Augustine Committee "Flexible Path" advocated developing a Human Spaceflight architecture that was capability focused rather than destination focus. This basic thought was captured in the Capability Driven 


\section{The Capability Driven Framework}

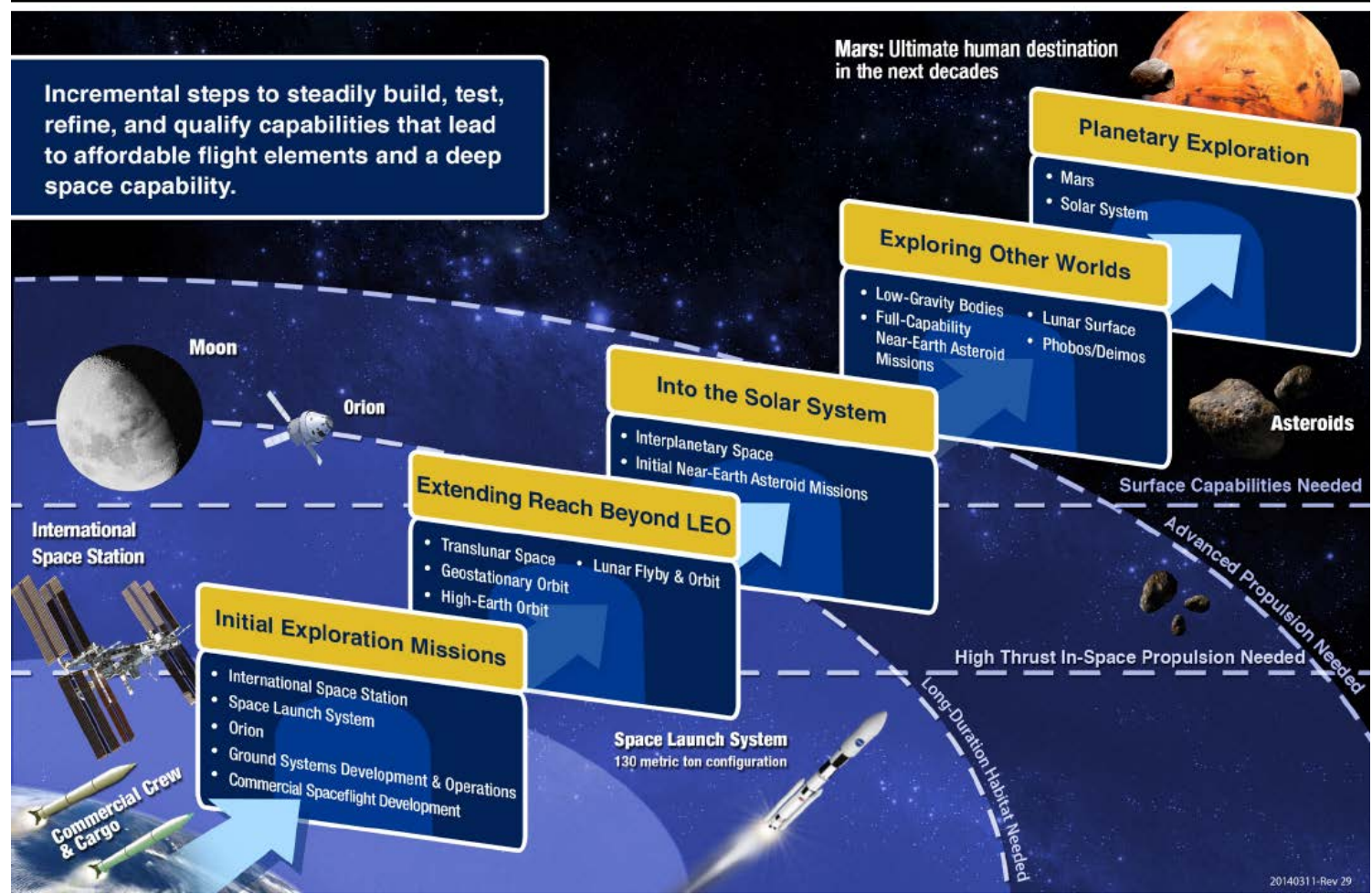

Figure 20. NASA's Capability Driven Framework

Framework by NASA in 2010, as shown in Fig. 20. The Capability Driven Framework (CDF) proposes developing enabling capabilities that could be utilized to implement any of many possible missions. The CDF then maps those basic capabilities to incremental destinations extending towards the ultimate goal of getting humans to the surface of Mars. The ARM technologies support the CDF as demonstrated in the previous sections. The SEP system developed for ARM could be utilized to support many other possible future missions. It could be part of a tug architecture supporting lunar missions, or its power level could be evolved to $100-250 \mathrm{~kW}$ to preposition assets for Mars missions. The AR\&D sensors directly support lunar, Mars, or other possible missions. The Orion and SLS systems enable "extending reach beyond LEO", but without the ARM redirected asteroid material there isn't a destination that would be reachable with those systems. The ARM mission reaches "into the solar system," performing an initial NEA mission. It also touches into "exploring other worlds" by giving operational experience working on low gravity bodies. Finally, the ARM extends human exploration by developing key technologies for future missions.

\section{Conclusion}

The ARM mission has great promise for extensibility. The SEP, AR\&D sensors and RBC Capture mission from the ARRV all play forward to support possible lunar or Mars moon missions. The docking system and EVA systems for the ARCM also generically support future missions. Thus, investment in the ARM concept provides logical stepping stones to future exploration goals.

\section{Acknowledgments}

The authors would like to acknowledge the support of the Asteroid Redirect Mission teams from JPL, LaRC, JSC, GRC, MSFC, GSFC, and KSC for their inputs, as well as Alberto G. Bertolin and Brad W. Reynolds from AMA Studios (a division of Analytical Mechanics Associates) in JSC. 


\section{References}

1“Rescue in Space”, Air Force Magazine, Vol. 95, No. 1, January 2012.

${ }^{2}$ Space Technology Mission Directorate website, Advanced Solar Array Systems webpage:

http://www.nasa.gov/offices/oct/home/feature_sas.html

${ }^{3}$ Asteroid Retrieval Feasibility Study, Keck Institute for Space Studies, http://www.kiss.caltech.edu/study/asteroid/asteroid_final_report.pdf, 2012.

${ }^{4}$ Asteroid Redirect Mission Reference Concept document, available at:

http://www.nasa.gov/pdf/756122main_Asteroid\%20Redirect\%20Mission\%20Reference\%20Concept\%20Description.pdf

${ }^{5}$ Brophy, John R., and Muirhead, Brian, “Near-Earth Asteroid Retrieval Mission (ARM) Study,” IEPC-2013-82, Presented at the $33^{\text {rd }}$ International Electric Propulsion Conference, Washington, DC, October 6-10, 2013.

${ }^{6}$ Brophy, John R., et al., "300-kW Solar Electric Propulsion System Configuration for Human Exploration of Near-Earth Asteroids,” AIAA2011-5514, 47 ${ }^{\text {th }}$ AIAA/ASME/SAE/ASEE Joint Propulsion Conference \& Exhibit, San Diego, CA, 21 July 03 August 2011.

${ }^{7}$ Strange, Nathan, et al, "Human Missions to Phobos and Deimos Using Combined Chemical and Solar Electric Propulsion", AIAA 2011-5663, 47th AIAA/ASME/SAE/ASEE Joint Propulsion Conference \& Exhibit, 31 July - 03 August 2011.

${ }^{8}$ Mercer, Carolyn, et al, “A Combined Solar Electric and Storable Chemical Propulsion Vehicle for Piloted Mars Missions”, AIAA SPACE 2013 Conference, 10-12 September 2013.

9,'The Office of Exploration FY1988 Annual Report,” NASA-TM-4075, December 1988.

${ }^{10}$ Griffin, Brand, et al, “A Comparison of Transportation Systems for Human Missions to Mars,” AIAA 2004-3834, 40th AIAA/ASME/SAE/ASEE Joint Propulsion Conference and Exhibit, 11 - 14 July 2004.

11,"The Office of Exploration FY1989 Annual Report," NASA-TM-4170, August 1989.

12”,The Global Exploration Roadmap,” produced by the International Space Exploration Coordination Group (ISECG), http://www.nasa.gov/sites/default/files/files/GER-2013_Small.pdf, August 2013. 